\title{
The predictive value of NT-proBNP and hs-TnT for risk of death in cardiac surgical patients
}

\section{Brynildsen, Jon}

2018-03

Brynildsen , J , Petäjä , L , Pettilä , V , Nygård , S, Vaara , S T , Linko , R , Okkonen , M , Hagve , T-A , Soininen, L , Suojaranta-Ylinen, R, Lyngbakken, M N , Omland , T \& Rosjo , $\mathrm{H} 2018$, ' The predictive value of NT-proBNP and hs-TnT for risk of death in cardiac surgical patients ' , Clinical Biochemistry , vol. 53 , pp. 65-71 . https://doi.org/10.1016/j.clinbiochem.2018.01.012

http://hdl.handle.net/10138/301255

https://doi.org/10.1016/j.clinbiochem.2018.01.012

publishedVersion

Downloaded from Helda, University of Helsinki institutional repository.

This is an electronic reprint of the original article.

This reprint may differ from the original in pagination and typographic detail.

Please cite the original version. 


\title{
The predictive value of NT-proBNP and hs-TnT for risk of death in cardiac surgical patients
}

\author{
Jon Brynildsen $^{\mathrm{a}, \mathrm{b}, 1}$, Liisa Petäjä ${ }^{\mathrm{c}, 1}$, Ville Pettilä ${ }^{\mathrm{d}, \mathrm{e}}$, Ståle Nygård ${ }^{\mathrm{f}}$, Suvi T. Vaara ${ }^{\mathrm{d}}$, Rita Linko ${ }^{\mathrm{d}}$, \\ Marjatta Okkonen $^{\mathrm{d}}$, Tor-Arne Hagve ${ }^{\mathrm{g}, \mathrm{h}}$, Leena Soininen ${ }^{\mathrm{d}}$, Raili Suojaranta-Ylinen ${ }^{\mathrm{c}}$, \\ Magnus Nakrem Lyngbakken ${ }^{\mathrm{a}, \mathrm{b}}$, Torbjørn Omland ${ }^{\mathrm{a}, \mathrm{b}}$, Helge Røsj $\varnothing^{\mathrm{a}, \mathrm{b}, *}$ \\ a Division of Medicine, Akershus University Hospital, Lørenskog, Norway \\ ${ }^{\mathrm{b}}$ Center for Heart Failure Research, Institute of Clinical Medicine, University of Oslo, Oslo, Norway \\ ${ }^{\mathrm{c}}$ Division of Anesthesiology, Department of Anesthesiology, Intensive Care and Pain Medicine, University of Helsinki, Helsinki University Hospital, Helsinki, Finland \\ ${ }^{\mathrm{d}}$ Division of Intensive Care Medicine, Department of Anesthesiology, Intensive Care and Pain Medicine, University of Helsinki, Helsinki University Hospital, Helsinki, \\ Finland \\ ${ }^{\mathrm{e}}$ Inselspital, Bern University Hospital, University of Bern, Bern, Switzerland \\ ${ }^{\mathrm{f}}$ Bioinformatics Core Facility, Institute for Medical Informatics, Oslo University Hospital, University of Oslo, Oslo, Norway \\ ${ }^{g}$ Division of Diagnostics and Technology, Akershus University Hospital, Lørenskog, Norway \\ ${ }^{\mathrm{h}}$ Institute of Clinical Medicine, University of Oslo, Oslo, Norway
}

\section{A R T I C L E I N F O}

\section{Keywords:}

Biomarkers

Cardiac surgery

Troponin T

NT-proBNP

Risk assessment

\begin{abstract}
A B S T R A C T
Background: European System for Cardiac Operative Risk Evaluation II (EuroSCORE II) is used for risk stratification before cardiac surgery, but whether N-terminal pro-B-type natriuretic peptide (NT-proBNP) and highsensitivity troponin T (hs-TnT) may add prognostic information to EuroSCORE II is not known.

Methods: Preoperative $(n=640)$ and postoperative $(n=629)$ blood samples were available from cardiac surgical patients with 961-day follow-up (FINNAKI Heart study; cohort \#1). The accuracy of a parsimonious risk model with NT-proBNP measurements was also tested in 90 patients with respiratory failure after cardiac surgery (FINNALI study; cohort \#2).

Results: Sixty-one patients $(9.5 \%)$ died during follow-up in cohort \#1. Preoperative NT-proBNP and hs-TnT concentrations correlated (rho $=0.58 ; \mathrm{p}<0.001$ ) and were higher in non-survivors compared to survivors: median 2027 (Q1-3 478-5387) vs. 373 (134-1354) ng/L [NT-proBNP] and 39 (16-191) vs. 13 (8-32) ng/L [hsTnT]; $\mathrm{p}<0.001$ for both. Preoperative NT-proBNP concentrations were associated with time to death after adjustment for EuroSCORE II (HR [ ${ }_{1 n}$ NT-proBNP] 1.33 [95\% CI 1.08-1.64]), p = 0.008 and reclassified patients on top of EuroSCORE II (net reclassification index 0.39 [95\% CI 0.14-0.64], p = 0.003). Pre- and postoperative NT-proBNP concentrations were closely correlated (rho $=0.80, \mathrm{p}<0.001$ ) and postoperative NT-proBNP concentrations were also associated with long-term mortality after adjustment for EuroSCORE II. A parsimonious risk model that included age, creatinine clearance, chronic pulmonary disease, and NT-proBNP measurements provided comparable prognostic accuracy as EuroSCORE II in cohort \#1 and \#2 for risk of long-term mortality. hs-TnT measurements did not add to NT-proBNP measurements

Conclusion: NT-proBNP measurements could improve and simplify risk prediction in cardiac surgical patients.
\end{abstract}

\section{Introduction}

Patients that are scheduled for cardiac surgery constitute a heterogeneous group with increased mortality compared to subjects of the general population [1-3]. Accordingly, the European System for Cardiac Operative Risk Evaluation (EuroSCORE) risk model was

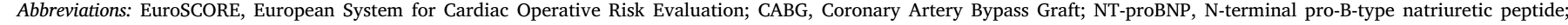

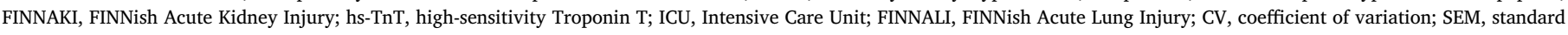

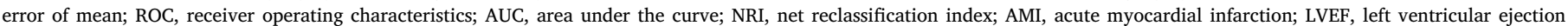
fraction; NYHA, New York Heart Association; CCS, Canadian Cardiovascular Society; IQR, inter quartile range; HR, hazard ratio; OR, odds ratio

* Corresponding author at: Division of Medicine, Akershus University Hospital, Sykehusveien 25, 1478 Lørenskog, Norway.

E-mail address: helge.rosjo@medisin.uio.no (H. Røsjø).

${ }^{1}$ These authors contributed equally to the work.
} 
developed to provide prognostic information concerning hospital mortality in cardiac surgical patients [4]. Later, this model has been revised as EuroSCORE II [5] and EuroSCORE II has also been validated to provide long-term risk prediction after isolated coronary artery bypass graft (CABG) surgery [6]. The EuroSCORE II score is calculated based on 18 variables, including age, gender and specific comorbidities, indices for functional status, estimated risk for the specific surgical procedure, and indices for cardiac function. Although recent myocardial infarction and measurements of left ventricular ejection fraction are included in EuroSCORE II to reflect cardiac status, circulating cardiac biomarkers are currently not included in risk models for cardiac surgical patients [5].

N-terminal pro-B-type natriuretic peptide (NT-proBNP) and highsensitivity troponin $\mathrm{T}$ (hs-TnT) are established cardiac biomarkers in routine clinical use in Europe [7,8]. NT-proBNP has an ability to discriminate between heart failure and non-cardiac dyspnea [9], while cardiac troponin is a sine qua non criterion to diagnose myocardial infarction in patients with acute coronary syndromes [7]. Circulating concentrations of NT-proBNP and hs-TnT have also been reported to provide incremental prognostic information to clinical risk indices across the spectrum of cardiovascular disease $[8,10]$, including in cardiac surgical patients [11-14] and in patients with intermediate EuroSCORE II risk score [15]. We have also recently reported that the combination of pre- and postoperative hs-TnT concentrations provided additional prognostic information to EuroSCORE II in patients with non-CABG cardiac surgery in the FINNish Acute Kidney Injury (FINNAKI) Heart Study [16]. Still, whether NT-proBNP or the combination of NT-proBNP and hs-TnT measurements adds prognostic information to EuroSCORE II, including reclassification, is currently not known. This information should be of clinical relevance as EuroSCORE II have suboptimal performance in high-risk patients and in patients subjected to isolated CABG surgery $[17,18]$, which supports the call for variables for a new and improved EuroSCORE III risk model [5]. Accordingly, in this study we hypothesized that (1) NT-proBNP and hs-TnT measurements would improve the EuroSCORE II risk model, as assessed by several statistical metrics, and (2) that a risk model with fewer key clinical variables and cardiac biomarkers (parsimonious risk model) could provide comparable prognostic information to the EuroSCORE II risk model.

\section{Methods}

\subsection{Cohort \#1: FINNAKI Heart Study}

The FINNAKI Heart Study is a single-center sub-study of the multicenter FINNAKI Study $[16,19]$. The FINNAKI Heart Study is a prospective observational study with an overall aim to evaluate the predictive value of cardiac biomarkers for all-cause mortality in patients subjected to cardiac surgery. The Ethics Committee in Helsinki University Hospital approved the study and informed consent was obtained from each patient or their next of kin. The flow chart of patient inclusion is presented in Fig. 1. All adult patients ( $\geq 18$ years) subjected to cardiac surgery in Helsinki University Hospital from September 1st 2011 to January 31st 2012 were eligible for inclusion into the study, except some predefined groups: patients planned for cardiac transplantation, patients that refused transfusions, and patients that were scheduled for implantation of ventricular assist devices or trans-femoral valve implantations. We also excluded patients that were not admitted to Intensive Care Units (ICUs) after surgery, non-Finnish residents, patients without informed consent, and patients without preoperative blood sampling.

Between February 1st 2012 and June 20th 2012 the FINNAKI Heart Study only included patients undergoing CABG. The endpoint of this study was all-cause mortality and maximum follow-up was 961 days. Demographic and clinical data were prospectively collected to the Finnish Intensive Care Consortium database (Tieto Ltd., Helsinki,

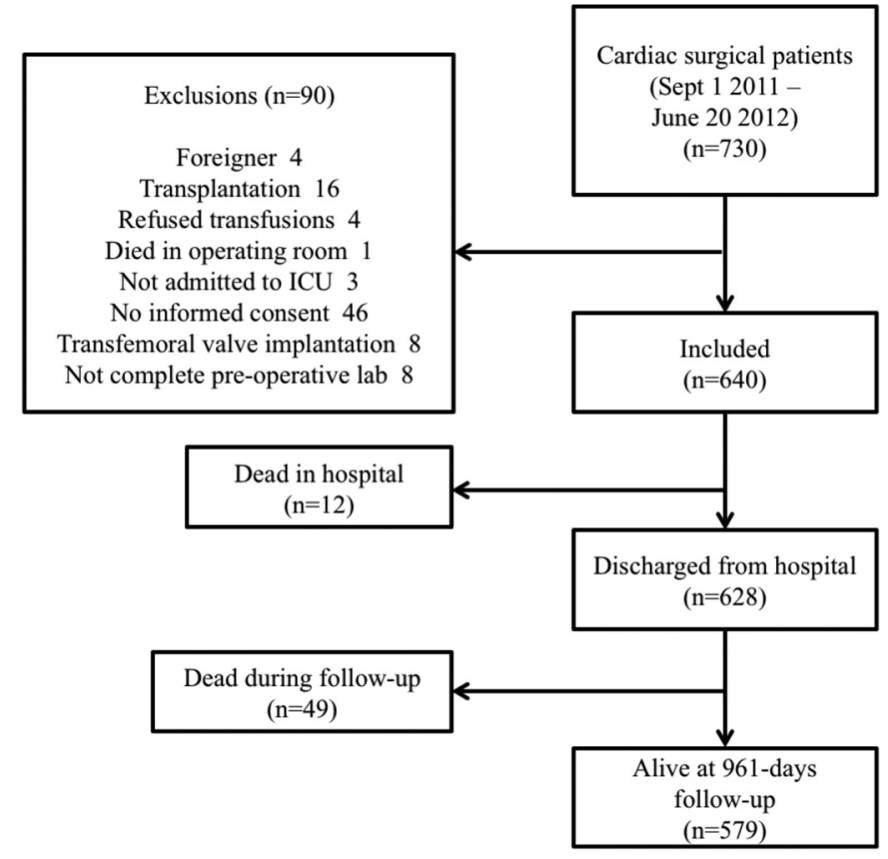

Fig. 1. Flowchart FINNAKI Heart Study (cohort \#1).

Finland) via study-specific case report forms and electronic anesthesia and intensive care patient reports (PICIS, Wakefield, MA, USA and Dräger Medical, Lübeck, Germany). Creatinine clearance was calculated according to the Cockcroft-Gault formula for EuroSCORE II [5]. Mortality data were obtained from the Finnish Population Register Centre and no patient was lost to follow-up.

\subsection{Cohort \#2: cardiac surgical patients in the FINNALI study}

The FINNish Acute Lung Injury (FINNALI) Study was a prospective observational multicenter study conducted during an 8-week period from April 16th 2007 to June 10th 2007, which enrolled patients treated for at least $6 \mathrm{~h}$ with ventilatory support in 25 Finnish ICUs (Supplementary Fig. 1) [20]. For this study, we included only patients from the FINNALI Study that underwent cardiac surgery prior to the need for ventilatory support and with blood sampling on ICU admission, entailing the five Finnish University Hospitals and Vasa Central Hospital (Supplementary Fig. 1; $\mathrm{n}=90$ ). Mean follow-up was 331 days ( \pm 10.69 days $[\mathrm{SEM}]$ ) and maximal follow-up was 365 days in cohort \#2. Data for all-cause mortality in cohort \#2 was obtained from Statistics Finland. We recorded information on comorbidities and standard laboratory variables from the patient chart records. The FINNALI Study was conducted according to the Declaration of Helsinki and the ethical consideration and approval from the Helsinki University Ethics Committee covered all hospitals included in the study. All patients or a close relative gave informed consent before study commencement.

\subsection{Blood sampling and biochemical analysis}

Blood samples in cohort \#1 were collected from arterial lines before induction of anesthesia and on the first postoperative morning. The samples were separated and stored at $-80^{\circ} \mathrm{C}$, and shipped on dry ice to Akershus University Hospital for hs-TnT and NT-proBNP analyses. Due to the inclusion strategy of cohort \#2 that required respiratory failure, only blood samples on day 1 after cardiac surgery were available in the FINNALI Study. These were stored for a short period at $-20{ }^{\circ} \mathrm{C}$ or colder in each of the participating hospitals before being moved to Helsinki University Central Hospital and stored at $-80{ }^{\circ} \mathrm{C}$ before NTproBNP measurements. In both studies the methods for storing blood 
samples have been shown to be adequate for stability in later analyses [21-23]. hs-TnT concentrations in cohort \#1 were determined on the auto-analyzer Cobas 8000 e801 (Roche Diagnostics) by a commercial assay (troponin T hs STAT, Roche Diagnostics). The lower detection limit is $5 \mathrm{ng} / \mathrm{L}$, limit of blank is $3 \mathrm{ng} / \mathrm{L}$, and the 99th percentile value in healthy subjects is $14 \mathrm{ng} / \mathrm{L}$ with a coefficient of variation (CV) of $8 \%$ for the hs-TnT assay [24,25]. Fifteen patients had missing preoperative hs$\mathrm{TnT}$ concentrations and one patient had hs-TnT concentration below the limit of blank and was assigned a concentration of $3 \mathrm{ng} / \mathrm{L}$. NTproBNP concentrations in cohort \#1 were measured by the commercially available immunometric assay (proBNP, Roche Diagnostics) on a Cobas 8000 e801 (Roche Diagnostics), while for cohort \#2 NT-proBNP concentrations were measured by a commercially available immunoassay (proBNP, Roche Diagnostics) on an Elecsys 2010 autoanalyzer (Roche Diagnostics) as previously described [26]. Specimens were run in one batch when analyzed.

\subsection{Statistics}

We report continuous variables with non-normal distribution as median (quartile 1-3), and categorical variables are presented as absolute number (percentage). We tested the distribution of continuous variables by the Kolmogorov-Smirnov test. To compare continuous variables we used the Mann-Whitney $U$ test and categorical data were compared by the Pearson Chi-Square test or the Fisher's exact test. Serial measurements were tested by the related-samples Wilcoxon signed rank test. We calculated correlation coefficients by Spearman rank correlation and explored variables associated with high preoperative hs-TnT and NT-proBNP concentrations by linear regression analyses.

To assess the association between preoperative hs-TnT and NTproBNP concentrations and time to death in cohort \#1, we stratified patients according to biomarker quartiles in Kaplan-Meier plots and compared the groups by the log-rank test. We also performed univariate and multivariate Cox proportional hazard regression analyses for follow-up data after discharge from hospital.

In the multivariate regression models regarding prognosis, all variables associated with mortality in the univariate analyses were included into the multivariate models (backward selection) except individual covariates of EuroSCORE II or variables covered by EuroSCORE II, as EuroSCORE II score was included in all multivariate analyses. For each variable that was included in the Cox multivariate analyses there were $>8$ events and thus overfitting should not be a problem in our analyses [27].

Prognostic accuracy was also assessed by receiver operating characteristic (ROC) analysis and presented as area under the curve (AUC) with $95 \%$ confidence intervals (CI). Data from nested models are presented as recently recommended [28]. We determined the optimal preand postoperative NT-proBNP concentration for assessing long-term mortality from ROC-AUC statistics and by the use of the Youden index J. We also explored whether preoperative hs-TnT and NT-proBNP concentrations, separately and in combination, could add to EuroSCORE II for the prediction of postoperative death by calculating the category-free net reclassification index (NRI) [29].

A parsimonious risk model means a model that has been reduced e.g. by stepwise elimination procedure, resulting in a model with a limited number of variables. To develop a parsimonious risk model for cardiac surgical patients, we first calculated ROC-AUC in cohort \#1 of all variables included in the EuroSCORE II individually, biomarkers individually, as well as the total EuroSCORE II regarding long-term mortality in the FINNAKI Heart Study (cohort \#1). Second, we arranged combinations of 2,3 , and 4 variables in cohort \#1 to identify the combination that gave the highest AUC using lasso regression [30]. We used the 4-unit combination with the highest AUC to create pre- and postoperative Cardiac Surgery Biomarker Scores and we tested the ROC-AUC of the postoperative Cardiac Surgery Biomarker Score in
Table 1

Baseline characteristics according to long-term survival status of cardiac surgical patients from the FINNAKI Heart Study (cohort \#1).

\begin{tabular}{|c|c|c|c|}
\hline & Survivors & Non-survivors & $P$ value \\
\hline & $\begin{array}{l}(\mathrm{N}=579 \\
90.5 \%)\end{array}$ & $(\mathrm{N}=61,9.5 \%)$ & \\
\hline Age (years) [0] & $67.0(59.0-74.0)$ & $75.0(65.5-80.0)$ & $<0.001$ \\
\hline Female sex [1] & $143(24.7 \%)$ & $18(29.5 \%)$ & 0.415 \\
\hline Body mass index $\left(\mathrm{m} / \mathrm{kg}^{2}\right)[0]$ & $27.1(24.2-30.5)$ & $25.4(23.2-30.4)$ & 0.09 \\
\hline LVEF (\%) $[0]$ & & & $<0.001$ \\
\hline$<21$ & $2(0.3 \%)$ & $2(3.3 \%)$ & \\
\hline $21-30$ & $19(3.3 \%)$ & $6(9.8 \%)$ & \\
\hline $31-50$ & $126(21.8 \%)$ & $19(31.1 \%)$ & \\
\hline$\geq 50$ & $432(74.6 \%)$ & $34(55.7 \%)$ & \\
\hline NYHA Functional Class [0] & & & 0.001 \\
\hline 4 & $139(24.0 \%)$ & $26(42.6 \%)$ & \\
\hline 3 & $207(35.8 \%)$ & $25(41.0 \%)$ & \\
\hline 2 & $187(32.3 \%)$ & $10(16.4 \%)$ & \\
\hline 1 & $46(7.9 \%)$ & $0(0.0 \%)$ & \\
\hline CCS Class 4 angina [0] & $174(30.2 \%)$ & $26(42.6 \%)$ & 0.044 \\
\hline $\begin{array}{l}\text { Estimated creatinine clearance } \\
\qquad(\mathrm{ml} / \mathrm{min})[3]\end{array}$ & $\begin{array}{l}83.2 \\
(66.2-109.4)\end{array}$ & $64.0(44.9-82.7)$ & $<0.001$ \\
\hline Smoking status $[6]$ & & & 0.51 \\
\hline Smoker & $84(14.6 \%)$ & $13(21.3 \%)$ & \\
\hline Ex-smoker & $126(21.9 \%)$ & $13(21.3 \%)$ & \\
\hline Non-smoker & $251(43.7 \%)$ & $21(34.4 \%)$ & \\
\hline Smoking status unknown & $112(19.5 \%)$ & $14(23 \%)$ & \\
\hline \multicolumn{4}{|l|}{ History of: } \\
\hline Hypertension [0] & $370(63.9 \%)$ & $45(73.8 \%)$ & 0.125 \\
\hline Heart failure [2] & $86(14.9 \%)$ & $21(34.4 \%)$ & $<0.001$ \\
\hline $\begin{array}{l}\text { Peripheral vascular disease } \\
\text { [0] }\end{array}$ & $88(15.2 \%)$ & $17(27.9 \%)$ & 0.011 \\
\hline Pulmonary disease [0] & $49(8.5 \%)$ & $14(23.0 \%)$ & $<0.001$ \\
\hline $\begin{array}{l}\text { Insulin-dependent diabetes } \\
\text { mellitus [0] }\end{array}$ & $52(9.0 \%)$ & $10(16.4 \%)$ & 0.063 \\
\hline $\begin{array}{l}\text { Non-insulin-dependent } \\
\text { diabetes mellitus [0] }\end{array}$ & $102(17.6 \%)$ & $8(13.1 \%)$ & 0.375 \\
\hline Recent AMI [0] & $187(32.3 \%)$ & $26(42.6 \%)$ & 0.104 \\
\hline Prior cardiac surgery [0] & $21(3.6 \%)$ & $3(4.9 \%)$ & 0.492 \\
\hline $\begin{array}{l}\text { Pulmonary hypertension } \\
\text { ( } \mathrm{mmHg})[0]\end{array}$ & & & $<0.001$ \\
\hline$<31$ & $378(65.3 \%)$ & $20(32.8 \%)$ & \\
\hline $31-55$ & $175(30.2 \%)$ & $28(45.9 \%)$ & \\
\hline$>55$ & $26(4.5 \%)$ & $13(21.3 \%)$ & \\
\hline Active endocarditis [0] & $9(1.6 \%)$ & $2(3.3 \%)$ & 0.283 \\
\hline Chronic renal disease [6] & $57(9.9 \%)$ & $15(25.0 \%)$ & $<0.001$ \\
\hline Chronic liver disease [3] & $3(0.5 \%)$ & $3(4.9 \%)$ & 0.001 \\
\hline Poor mobility [0] & $10(1.7 \%)$ & $4(6.6 \%)$ & 0.036 \\
\hline Urgency: [0] & & & 0.003 \\
\hline Elective & $350(60.4 \%)$ & $29(47.5 \%)$ & \\
\hline Urgent & $189(32.6 \%)$ & $23(37.7 \%)$ & \\
\hline Emergency & $40(6.9 \%)$ & $8(13.1 \%)$ & \\
\hline Salvage & $0(0.0 \%)$ & $1(1.6 \%)$ & \\
\hline $\begin{array}{l}\text { Cardiogenic shock at } \\
\text { admission/prior to } \\
\text { surgery [0] }\end{array}$ & $11(1.9 \%)$ & $5(8.2 \%)$ & $<0.003$ \\
\hline $\begin{array}{l}\text { Assisted vent. in use before } \\
\text { surgery [5] }\end{array}$ & $24(4.2 \%)$ & $10(16.7 \%)$ & $<0.001$ \\
\hline Intra-aortic balloon pump [5] & $14(2.4 \%)$ & $2(3.3 \%)$ & 0.657 \\
\hline Critical preoperative state $[0]$ & $40(6.9 \%)$ & $13(21.3 \%)$ & $<0.001$ \\
\hline Weight of intervention [0] & & & 0.11 \\
\hline Isolated CABG & $383(66.1 \%)$ & $33(54.1 \%)$ & \\
\hline Single non-CABG & $98(16.9 \%)$ & $14(23.0 \%)$ & \\
\hline Two procedures & $79(13.6 \%)$ & $9(14.8 \%)$ & \\
\hline Three or more procedures & $19(3.3 \%)$ & $5(8.2 \%)$ & \\
\hline \multicolumn{4}{|l|}{ Preoperative values: } \\
\hline hs-TnT (ng/L) [15] & $13(8-32)$ & $39(16-191)$ & $<0.001$ \\
\hline NT-proBNP (ng/L) [0] & $373(134-1354)$ & $\begin{array}{l}2027 \\
(478-5387)\end{array}$ & $<0.001$ \\
\hline EuroSCORE II (\%) [0] & $2.1(1.2-4.1)$ & $6.7(2.9-15.8)$ & $<0.001$ \\
\hline \multicolumn{4}{|l|}{ Perioperative values: } \\
\hline $\begin{array}{l}\text { Aortic occlusion time (min) } \\
{[0]}\end{array}$ & $65.0(50.0-90.0)$ & $59.0(35.5-82.0)$ & 0.07 \\
\hline $\begin{array}{l}\text { Cardio-pulmonary-bypass } \\
\text { time (min) [0] }\end{array}$ & $\begin{array}{l}93.0 \\
(72.0-125.0)\end{array}$ & $\begin{array}{l}90.0 \\
(69.5-120.5)\end{array}$ & 0.83 \\
\hline
\end{tabular}


Table 1 (continued)

\begin{tabular}{|c|c|c|c|}
\hline & Survivors & Non-survivors & $P$ value \\
\hline & $\begin{array}{l}(\mathrm{N}=579 \\
90.5 \%)\end{array}$ & $(\mathrm{N}=61,9.5 \%)$ & \\
\hline $\begin{array}{l}\text { Surgery on thoracic aorta } \\
\text { [1] }\end{array}$ & $37(6.4 \%)$ & $1(1.7 \%)$ & 0.24 \\
\hline \multicolumn{4}{|l|}{ Postoperative values: } \\
\hline hs-TnT (ng/L) [11] & $439(267-866)$ & $576(296-1182)$ & 0.09 \\
\hline NT-proBNP (ng/L) [11] & $\begin{array}{l}1269 \\
(646-2559)\end{array}$ & $\begin{array}{l}2913 \\
(1213-8951)\end{array}$ & $<0.001$ \\
\hline
\end{tabular}

AMI, Acute Myocardial Infarction; CABG, Coronary Artery Bypass Graft; CCS Class 4, Canadian Cardiovascular Society Class 4; EuroSCORE II, European System for Cardiac Operative Risk Evaluation II; hs-TnT, high-sensitivity Troponin T; LVEF, Left Ventricular Ejection Fraction; NT-proBNP, N-terminal pro-B-type natriuretic peptide; NYHA, New York Heart Association.

Continuous variables are presented as median (IQR). Categorical variables are presented as counts (percentage).

Numbers of patients with missing data in brackets.

cohort \#2 for external validation. Data were considered significant with a two-sided $\mathrm{P}$ value of $<0.05$. Statistical analyses were performed using IBM SPSS Statistics 22 and 24 (IBM Corp, Armonk, NY, USA), MedCalc Statistical Software version 14.10.2 (MedCalc Software, Ostend, Belgium), and the statistical programming language R (R Foundation for Statistical Computing, Vienna, Austria).

\section{Results}

\subsection{FINNAKI Heart Study: baseline characteristics}

Of the 640 patients included in cohort \#1, 416 patients (65\%) underwent isolated CABG, 112 patients (17.5\%) single non-CABG surgery, 88 patients $(13.8 \%)$ underwent two procedures, and 24 patients $(3.8 \%)$ were subjected to three or more procedures (Supplementary Table 1). In total, 61 patients (9.5\%) died within 961 days after surgery (Fig. 1). Baseline characteristics with patients stratified according to mortality are presented in Table 1 . Non-survivors in cohort \#1 were older, had more frequent and severe cardio-pulmonary disease, reduced renal function, and more often underwent acute surgery compared to patients surviving beyond 961 days after surgery. EuroSCORE II, preoperative NT-proBNP and hs-TnT concentrations, and postoperative NT-proBNP concentrations were all significantly higher in long-term non-survivors compared to survivors. Type of surgical procedure did not differ between long-term survivors and non-survivors (Supplementary Table 1; $\mathrm{p}=0.11$ ).

\subsection{Cardiac biomarker concentrations in cardiac surgical patients: cohort} \#1

Preoperative NT-proBNP and hs-TnT concentrations correlated (rho $=0.58 ; \mathrm{p}<0.001$ ) and medians [quartile 1-3] were 439 (148-1574) ng/L and 15 (8-37) ng/L, respectively, in the FINNAKI Heart Study (cohort \#1). In multivariate linear regression analyses that explained $61 \%$ of the variance in preoperative NT-proBNP concentrations $\left(r^{2}=0.61\right)$, several factors were associated with high NT-proBNP concentrations, including critical preoperative state and the weight of the intervention (CABG vs. other cardiac surgery) (Supplementary Table 2). High preoperative hs-TnT concentrations were also associated with a number of variables in multivariate linear regression analysis, including recent acute myocardial infarction, surgical urgency, critical preoperative state, weight of the intervention, and preoperative NTproBNP concentration $\left(\mathrm{r}^{2}=0.58\right.$; Supplementary Table 3$)$.

Postoperative NT-proBNP and hs-TnT concentrations in the FINNAKI Heart Study were 1352 (677-2853) ng/L and 451 (268-894) ng/L, respectively. Both values were significantly higher than
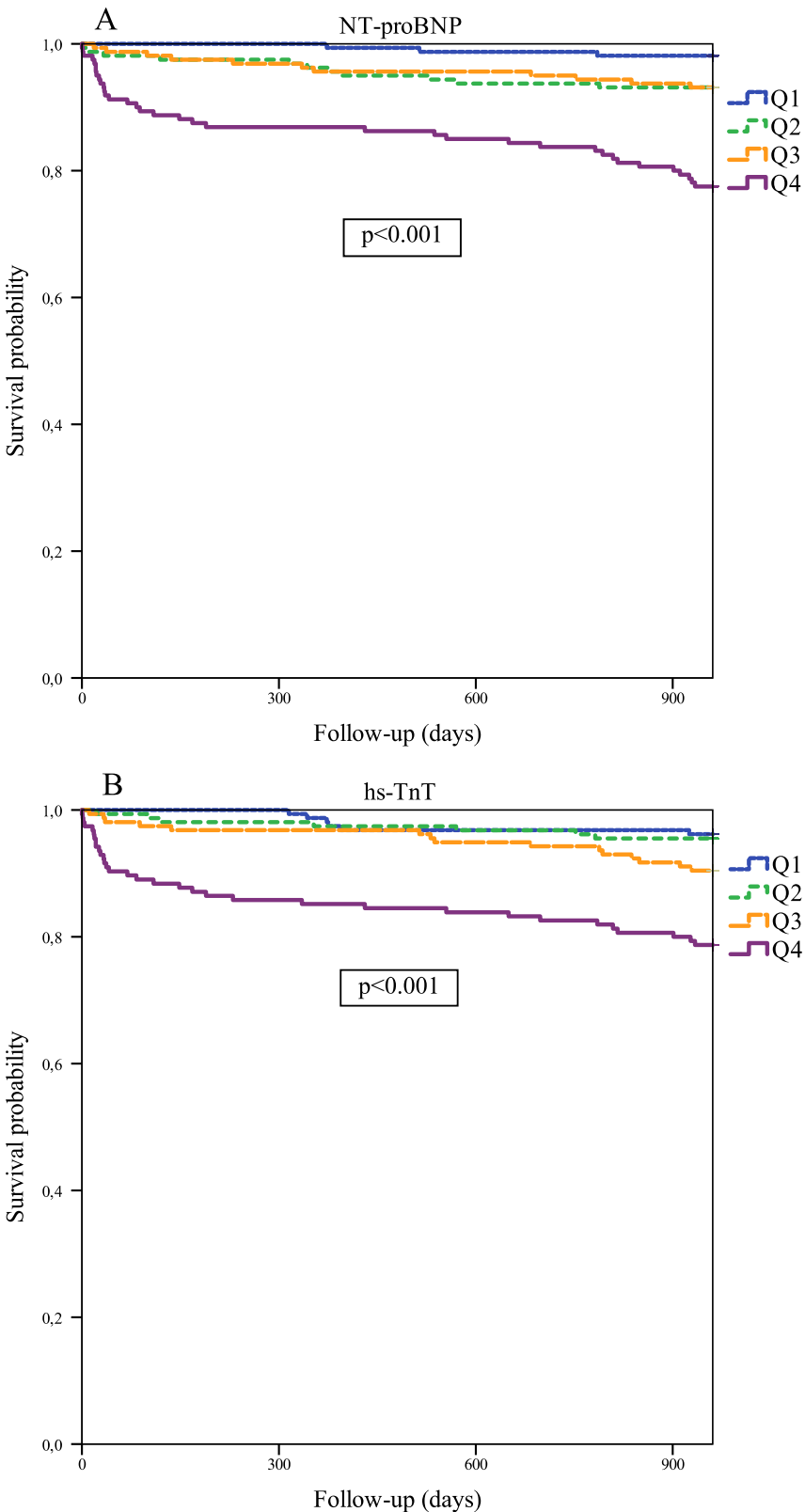

Fig. 2. Kaplan-Meier survival plots divided by quartiles of (A) preoperative NT-proBNP concentrations (ng/L) [Q1 < 148, Q2 148-438, Q3 439-1574, Q4 > 1574] and (B) hsTnT concentrations (ng/L) [Q1 < 8.1, Q2 8.1-14.7, Q3 14.7-37.1, Q4 > 37.1].

preoperative concentrations ( $\mathrm{p}<0.001$ ). We found significant correlations between pre- and postoperative concentrations for both NTproBNP $\quad(r h o=0.80, \quad \mathrm{p}<0.001) \quad$ and hs-TnT $\quad($ rho $=0.27$, $\mathrm{p}<0.001$ ), and postoperative NT-proBNP and hs-TnT concentrations were also correlated (rho $=0.42, \mathrm{p}<0.001$ ). Duration of cardiac surgery and some of the same clinical variables that influenced preoperative measurements were also associated with postoperative NTproBNP and hs-TnT concentrations (Supplementary Tables 4 and 5).

\subsection{FINNAKI Heart Study: cardiac biomarker concentrations and mortality}

Kaplan-Meier plots with biomarker concentrations divided into quartiles, NT-proBNP (ng/L) [Q1 < 148, Q2 148-438, Q3 439-1574, Q4 > 1574] and hs-TnT (ng/L) [Q1 < 8.1, Q2 8.1-14.7, Q3 14.7-37.1, Q4 > 37.1], demonstrate that patients with preoperative NT-proBNP and hs-TnT concentrations in the 4th quartile had increased mortality during 961-day follow-up (Fig. 2; p $<0.001$ with the log- 
Table 2

Predictive factors for time to death in in 961 days by univariate and multivariate Cox proportional hazard regression.

\begin{tabular}{|c|c|c|c|c|}
\hline & \multicolumn{4}{|c|}{961 days } \\
\hline & HR & $95 \% \mathrm{CI}$ & $\mathrm{P}$ value & Wald \\
\hline \multicolumn{5}{|l|}{ Univariate analysis } \\
\hline Age (per one year) ${ }^{a}$ & 1.07 & $1.04-1.10$ & $<0.001$ & 21.7 \\
\hline Female sex ${ }^{a}$ & 1.27 & $0.73-2.20$ & 0.40 & 0.7 \\
\hline Body mass index $\left(\mathrm{m} / \mathrm{kg}^{2}\right)$ & 0.96 & $0.91-1.02$ & 0.18 & 1.8 \\
\hline LVEF $(\geq 50 \%)^{\mathrm{a}}$ & 0.45 & $0.27-074$ & 0.002 & 9.8 \\
\hline NYHA functional class $(\geq 3)^{\mathrm{a}}$ & 3.30 & $1.67-6.49$ & 0.001 & 11.8 \\
\hline CCS class 4 angina $^{\mathrm{a}}$ & 1.69 & $1.02-2.81$ & 0.042 & 4.1 \\
\hline $\begin{array}{l}\text { Estimated creatinine clearance (per } \\
\text { one unit) }{ }^{\mathrm{a}, \mathrm{b}}\end{array}$ & 0.21 & $0.12-0.36$ & $<0.001$ & 31.1 \\
\hline Smoking (current/ex-smoker) & 1.27 & $0.77-2.11$ & 0.36 & 0.9 \\
\hline \multicolumn{5}{|l|}{ History of: } \\
\hline Hypertension & 1.56 & $0.88-2.76$ & 0.13 & 2.3 \\
\hline Heart failure & 2.81 & $1.66-4.77$ & $<0.001$ & 14.7 \\
\hline Peripheral vascular disease $^{\mathrm{a}}$ & 2.07 & $1.18-3.62$ & 0.011 & 6.5 \\
\hline Pulmonary disease $^{a}$ & 2.96 & $1.63-5.39$ & $<0.001$ & 12.7 \\
\hline Insulin-dependent diabetes mellitus ${ }^{\mathrm{a}}$ & 1.91 & $0.97-3.76$ & 0.061 & 3.5 \\
\hline $\begin{array}{l}\text { Non-insulin-dependent diabetes } \\
\text { mellitus }\end{array}$ & 0.71 & $0.34-1.49$ & 0.36 & 0.8 \\
\hline Recent $\mathrm{AMI}^{\mathrm{a}}$ & 1.54 & $0.93-2.55$ & 0.10 & 2.8 \\
\hline Previous cardiac surgery $^{\mathrm{a}}$ & 1.37 & $0.43-4.37$ & 0.60 & 0.3 \\
\hline \multicolumn{5}{|l|}{ Pulmonary arterial pressure $(\mathrm{mmHg})^{a}$} \\
\hline $31-55^{c}$ & 2.91 & $1.64-5.17$ & $<0.001$ & 13.4 \\
\hline$>55^{c}$ & 7.84 & $3.90-15.8$ & $<0.001$ & 33.4 \\
\hline Active endocarditis ${ }^{\mathrm{a}}$ & 2.14 & $0.52-8.77$ & 0.29 & 1.1 \\
\hline Chronic renal disease & 2.73 & $1.52-4.90$ & $<0.001$ & 11.4 \\
\hline Chronic liver disease & 7.03 & $2.20-22.48$ & 0.001 & 10.8 \\
\hline Poor mobility $^{\mathrm{a}}$ & 3.64 & $1.32-10.05$ & 0.012 & 6.2 \\
\hline Urgency (elective vs acute) ${ }^{\mathrm{a}}$ & 1.65 & $0.999-2.73$ & 0.05 & 3.8 \\
\hline $\begin{array}{l}\text { Cardiogenic shock at admission/prior } \\
\text { to surgery }\end{array}$ & 4.07 & $1.63-10.16$ & 0.003 & 9.0 \\
\hline Assisted vent. in use before surgery & 4.21 & $2.13-8.30$ & $<0.001$ & 17.2 \\
\hline Intra-aortic balloon pump & 1.38 & $0.34-5.65$ & 0.66 & 0.2 \\
\hline Critical preoperative state $^{\mathrm{a}}$ & 3.46 & $1.87-6.38$ & $<0.001$ & 15.7 \\
\hline $\begin{array}{l}\text { Weight of intervention } \\
\text { (isolated CABG vs other card } \\
\text { surg) }^{\text {a }}\end{array}$ & 0.63 & $0.38-1.05$ & 0.08 & 3.1 \\
\hline \multicolumn{5}{|l|}{ Preoperative values: } \\
\hline hs-TnT (per one unit) ${ }^{\mathrm{b}}$ & 1.34 & $1.20-1.51$ & $<0.001$ & 25.6 \\
\hline NT-proBNP (per one unit) ${ }^{b}$ & 1.68 & $1.43-1.97$ & $<0.001$ & 41.0 \\
\hline EuroSCORE II (per one unit) $^{\mathrm{b}}$ & 2.13 & $1.73-2.62$ & $<0.001$ & 50.9 \\
\hline \multicolumn{5}{|l|}{ Perioperative values: } \\
\hline Aortic occlusion time (min) & 0.995 & $0.988-1.002$ & 0.16 & 2.0 \\
\hline Cardio-pulmonary-bypass time (min) & 1.00 & $0.996-1.005$ & 0.94 & 0.01 \\
\hline Surgery on thoracic aorta ${ }^{a}$ & 0.26 & $0.04-1.84$ & 0.18 & 1.8 \\
\hline \multicolumn{5}{|l|}{ Multivariate analysis: $N T$-proBNP $P^{d}$} \\
\hline EuroSCORE II (per one unit) $^{\mathrm{b}}$ & 1.63 & $1.24-2.16$ & 0.001 & 11.92 \\
\hline NT-proBNP (per one unit) ${ }^{b}$ & 1.32 & $1.07-1.63$ & 0.009 & 6.83 \\
\hline
\end{tabular}

AMI, Acute Myocardial Infarction; CABG, Coronary Artery Bypass Graft; CCS Class 4, Canadian Cardiovascular Society Class 4; CI, Confidence Interval; EuroSCORE II, European System for Cardiac Operative Risk Evaluation II; hs-TnT, high-sensitivity Troponin T; HR, Hazard Ratio; LVEF, Left Ventricular Ejection Fraction; NT-proBNP, Nterminal pro-B-type natriuretic peptide; NYHA, New York Heart Association.

${ }^{\text {a }}$ Part of the EuroSCORE II calculations.

b Estimated creatinine clearance, NT-proBNP, hs-TnT and EuroSCORE II were transformed with natural logarithm before regression analysis because of non-normal distribution.

c Compared to $<31 \mathrm{~mm} \mathrm{Hg}$.

${ }^{\mathrm{d}}$ Variables retained in the final model with preoperative NT-proBNP and preoperative variables not included in the EuroSCORE II model or covered by variables in ESII model $(-2 \log$ likelihood $=727)$.

rank test for both). In univariate Cox regression analyses, both preoperative NT-proBNP and hs-TnT concentrations were significantly associated with time to death in 961 days, while only preoperative NTproBNP concentrations provided additional prognostic information to EuroSCORE II (Table 2). The optimal preoperative NT-proBNP concentration for assessing long-term risk was $1170 \mathrm{ng} / \mathrm{L}$ with sensitivity 66\% (95\% CI 52-77\%), specificity 73\% (69-77\%), positive likelihood ratio $2.5(2.0-3.1)$ and negative likelihood ratio 0.5 (0.3-0.7). For postoperative NT-proBNP the optimal cut-off was $1834 \mathrm{ng} / \mathrm{L}$ with sensitivity $70 \%(56-81 \%)$, specificity $66 \%(62-70 \%)$, positive likelihood ratio 2.1 (1.7-2.5), and negative likelihood ratio 0.5 (0.3-0.7).

Exploring hs-TnT and NT-proBNP in separate multivariate Cox regression analyses did not change the results (Supplementary Table 6). The ROC-AUC of preoperative NT-proBNP concentrations to predict 961-day mortality was 0.734 (95\% CI $0.668-0.800$ ), the AUC of EuroSCORE II was $0.740(0.672-0.807)$, and the AUC of preoperative NT-proBNP and EuroSCORE II was 0.762 (0.699-0.826). Preoperative NT-proBNP concentrations also reclassified a significant proportion of patients when added to EuroSCORE II with 388 patients being moved to a more correct risk stratum (61\%), while NT-proBNP measurements failed to improve risk assessment in 252 patients: NRI $=0.39$ (95\% CI 0.14-0.64), $\mathrm{p}=0.003$. Hence, preoperative NT-proBNP concentrations moved patients that did not experience an event down in risk stratum and moved patients that experienced an event up in risk stratum when added on top of EuroSCORE II (Fig. 3). In contrast, the addition of hsTnT to EuroSCORE II did not improve risk prediction as tested by NRI $(0.09[-0.11-0.29, \mathrm{p}=0.37])$ and the AUC of preoperative hs-TnT measurements was $0.700(0.631-0.769)$.

Assessing also postoperative cardiac biomarker concentrations, only NT-proBNP concentrations were associated with long-term mortality when measured day 1 after cardiac surgery (Table 1 and Supplementary Fig. 2). The ROC-AUC of postoperative NT-proBNP concentrations to separate long-term survivors from non-survivors was 0.711 (0.693-0.782) and the AUC of postoperative hs-TnT concentrations was 0.566 (0.485-0.648). Postoperative NT-proBNP concentrations were also significantly associated with long-term outcome in multivariate analysis that included EuroSCORE II, while postoperative hs-TnT did not provide additional prognostic information to EuroSCORE II in the total cohort (Supplementary Table 7).

\subsection{Cardiac surgery biomarker score: parsimonious risk model}

Twelve of the eighteen variables included in the EuroSCORE II calculations were associated with mortality in the FINNAKI Heart Study when analyzed separately (cohort \#1; Supplementary Table 8). The 4variable combination with the highest ROC-AUC for 961-day mortality in cohort \#1 included (1) preoperative NT-proBNP concentrations, (2) age, (3) estimated creatinine clearance, and (4) history of chronic pulmonary disease. This parsimonious Cardiac Surgery Biomarker Score yielded an AUC of 0.787 (0.726-0.848) for all-cause mortality in cohort \#1. The corresponding AUC of EuroSCORE II for 961-day mortality was 0.744 (0.676-0.811). Calculating the AUC for the Cardiac Surgery Biomarker Score using postoperative instead of preoperative NT-proBNP yielded an AUC of $0.766(0.699-0.832)$ in cohort \#1 and an AUC of 0.819 (0.613-1.00) for all-cause mortality in cohort \#2. Baseline characteristics of cardiac surgical patients in cohort \#2 are presented in Supplementary Table 9.

\section{Discussion}

In this prospective observational study comprising two separate cohorts of critically ill patients after cardiac surgery we found that NTproBNP measurements had predictive value for time to death. A 4-item Cardiac Surgery Biomarker Score including NT-proBNP measurements also had at least comparable discriminative value as the 18-item EuroSCORE II for long-term risk of death.

The original EuroSCORE was created as a risk prediction tool for hospital mortality following cardiac surgery [4]. However, as the majority of cardiac surgical patients die after hospital discharge [2], the original EuroSCORE was later proven useful also to predict long-term mortality [31,32]. EuroSCORE II was subsequently developed and found superior to the original EuroSCORE risk model [33,34]. However, there are some obvious limitations to EuroSCORE II, including the 

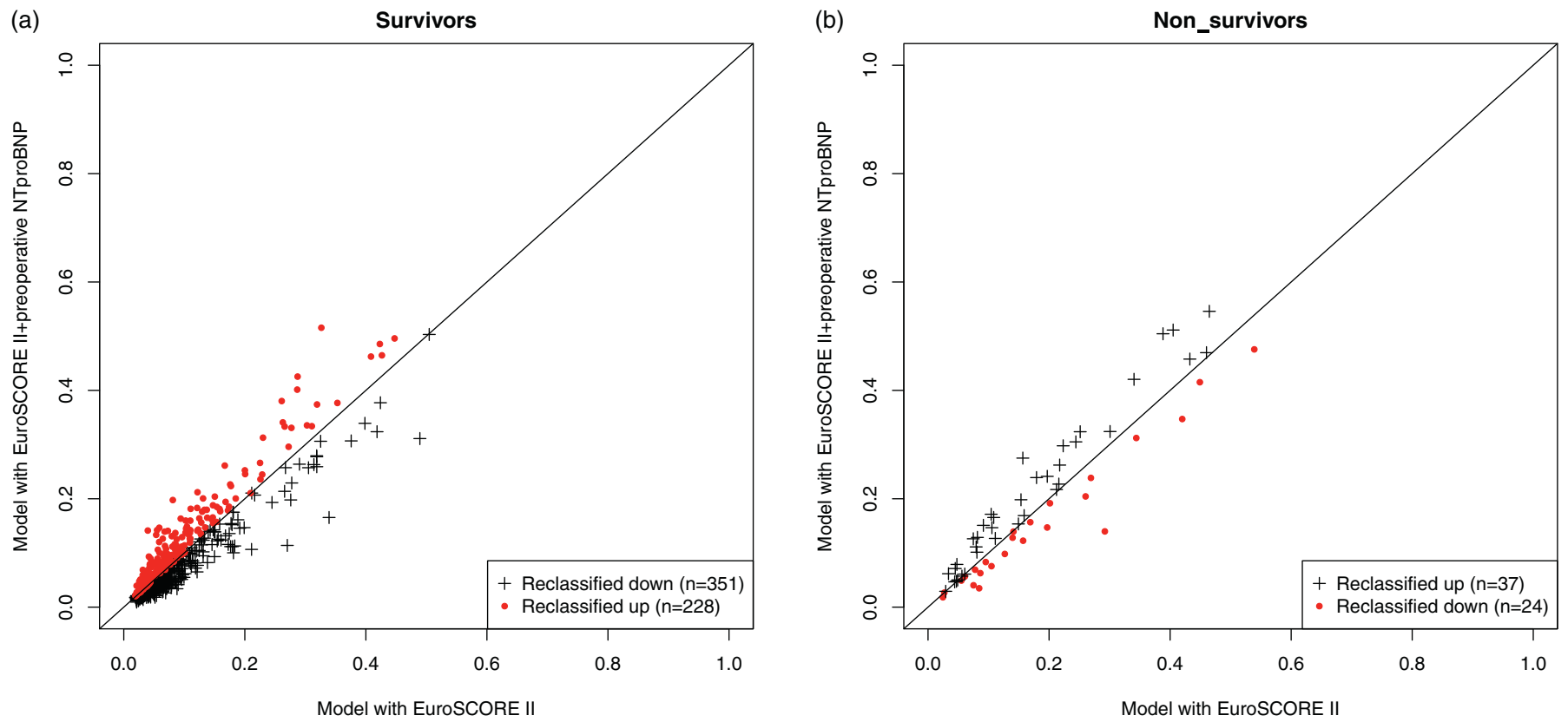

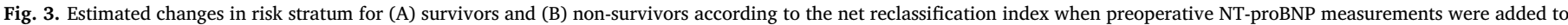
EuroSCORE II.

need to collect data on 18 variables. Moreover, EuroSCORE II also includes invasive pulmonary pressure measurements, which limits the usefulness of this model for preoperative risk assessment. EuroSCORE II is neither validated for use in patients older than 90 years [5], and some recommend that EuroSCORE II should not be used in patients over 70 years [35]. Recent studies have also questioned the accuracy of EuroSCORE II in patients subjected to isolated CABG and in high-risk patient groups $[17,18]$.

Cardiac biomarkers have obtained a strong position in clinical cardiology, including the potential of NT-proBNP to improve risk prediction in cardiac surgical patients [11-13,15]. We now validate and extend these previous data by demonstrating that pre- and postoperative NT-proBNP measurements provide incremental prognostic information to EuroSCORE II in cardiac surgical patients. Accordingly; we believe NT-proBNP should be included in a revised EuroSCORE III risk model. Pertinent to this point; we also demonstrate that a 4-item Biomarker Risk Score of age, estimated creatinine clearance, history of chronic pulmonary disease, and NT-proBNP measurements provide at least comparable prognostic information as the 18-item EuroSCORE II for long-term risk prediction in cardiac surgical patients. Thus, although this Biomarker Risk Score will need validation in other external cohorts, our data suggest that NT-proBNP measurements could also simplify risk assessment in cardiac surgical patients. As NT-proBNP primarily is considered a marker of cardiomyocyte stress and heart failure development, it is possible also that stringent postoperative control of subjects with high NT-proBNP concentration could help reduce heart failure-related mortality during follow-up in cardiac surgical patients. In contrast, hs-TnT measurements did not improve risk assessment on top of EuroSCORE II in our cardiac surgical patients. Of note, perioperative NT-proBNP measurement is also recommended to improve risk assessment in selected groups of patients undergoing noncardiac surgery [36]. We have not directly tested whether BNP measurements would have provided similar results as NT-proBNP in our study, but in general these two assays for pro-B-type natriuretic peptide measurement are considered equal [37] and it is plausible that also BNP measurements would have added prognostic information to EuroSCORE II in our cohort.

\subsection{Strengths and limitations}

This study has some limitations and strengths. First, the FINNAKI Heart Study only included patients from only one tertiary cardiac surgery center and therefore our data will need validation in additional cohorts. Second, we included different types of cardiac surgical patients into these analyses, although the majority of patients were subjected to CABG. Finally, external validation of preoperative NT-proBNP measurements is also needed for the Cardiac Surgery Biomarker Score, however, it is promising that it performed well with postoperative NTproBNP in cohort \#2. Potential strengths of the study include the use of contemporary biomarker assays, comprehensive statistical analyses according to established guidelines for biomarker studies [38], and benchmarking with the EuroSCORE II model.

\section{Conclusion}

We found that pre- and postoperative NT-proBNP measurements add to EuroSCORE II for risk prediction in cardiac surgical patients. A 4item Cardiac Surgery Biomarker Score with NT-proBNP measurements could also simplify long-term risk prediction in cardiac surgical patients.

\section{Acknowledgements}

We are thankful for the contribution by the personnel in all the participating centers in the FINNAKI Study, the FINNAKI Heart Study, and the FINNALI Study.

\section{Conflict of interest}

JB, LP, VP, SN, RL, MO, TAH, LS, RS, MN: These authors declare that they have no competing interests. STV: Been a member of the editorial board of the Acta Anaesthesiologica Scandinavia since Oct 2016. TO: Served on advisory boards and received speaker's honoraria and travel funding from Roche Diagnostics. He has also received research support via Akershus University Hospital.

HR: Roche Diagnostics (Penzberg, Germany) provided hs-TnT and NT-proBNP kits at a reduced price via Akershus University Hospital. 


\section{Funding}

This work was supported by Akershus University Hospital. The FINNAKI Study got EVO grants from Helsinki University Hospital [grant numbers TYH 2013343, TYH 2013106, T102010070], and from the Academy of Finland. The FINNALI study got EVO grants from Helsinki University Hospital [grant numbers TYH 7250, TYH 8240] and the Instrumentarium Scientific Foundation. The sponsor had no role in any of the following: design and conduct of the study, collection, management, analysis and interpretation of the data, or preparation, review and approval of the manuscript.

\section{Appendix A. Supplementary data}

Supplementary data to this article can be found online at https:// doi.org/10.1016/j.clinbiochem.2018.01.012.

\section{References}

[1] F. Roques, S.A. Nashef, P. Michel, E. Gauducheau, C. de Vincentiis, E. Baudet, J. Cortina, M. David, A. Faichney, F. Gabrielle, E. Gams, A. Harjula, M.T. Jones, P.P. Pintor, R. Salamon, L. Thulin, Risk factors and outcome in European cardiac surgery: analysis of the EuroSCORE multinational database of 19030 patients, Eur. J. Cardiothorac. Surg. 15 (6) (1999) 816-822 discussion 822-3 https://doi.org/10. 1016/S1010-7940(99)00106-2.

[2] S. Siregar, R.H. Groenwold, B.A. de Mol, R.G. Speekenbrink, M.I. Versteegh, G.J. Brandon Bravo, M.L. Bots Bruinsma, Y. van der Graaf, L.A. van Herwerden, Evaluation of cardiac surgery mortality rates: 30-day mortality or longer follow-up? Eur. J. Cardiothorac. Surg. 44 (5) (2013) 875-883, http://dx.doi.org/10.1093/ ejcts/ezt119.

[3] United Nations, Department of Economic and Social Affairs, Population Division, World Mortality Report 2013, United Nations publication, 2013.

[4] S.A. Nashef, F. Roques, P. Michel, E. Gauducheau, S. Lemeshow, R. Salamon, European system for cardiac operative risk evaluation (EuroSCORE), Eur. J. Cardiothorac. Surg. 16 (1) (1999) 9-13, http://dx.doi.org/10.1016/S10107940(99)00134-7.

[5] S.A. Nashef, F. Roques, L.D. Sharples, J. Nilsson, C. Smith, A.R. Goldstone, U. Lockowandt, EuroSCORE II, Eur. J. Cardiothorac. Surg. 41 (4) (2012) 734-744 discussion 744-5 https://doi.org/10.1093/ejcts/ezs043.

[6] F. Biancari, F. Vasques, R. Mikkola, M. Martin, J. Lahtinen, J. Heikkinen, Validation of EuroSCORE II in patients undergoing coronary artery bypass surgery, Ann. Thorac. Surg. 93 (6) (2012) 1930-1935, http://dx.doi.org/10.1016/j.athoracsur. 2012.02.064.

[7] K. Thygesen, J.S. Alpert, A.S. Jaffe, M.L. Simoons, B.R. Chaitman, H.D. White, et al., Third universal definition of myocardial infarction, Eur. Heart J. 33 (20) (2012) 2551-2567, http://dx.doi.org/10.1093/eurheartj/ehs184.

[8] L.B. Daniels, A.S. Maisel, Natriuretic peptides, J. Am. Coll. Cardiol. 50 (25) (2007) 2357-2368, http://dx.doi.org/10.1016/j.jacc.2007.09.021.

[9] J.L. Januzzi Jr., C.A. Camargo, S. Anwaruddin, A.L. Baggish, A.A. Chen, D.G. Krauser, R. Tung, R. Cameron, J.T. Nagurney, C.U. Chae, D.M. Lloyd-Jones, D.F. Brown, S. Foran-Melanson, P.M. Sluss, E. Lee-Lewandrowski, K.B. Lewandrowski, The N-terminal Pro-BNP investigation of dyspnea in the emergency department (PRIDE) study, Am. J. Cardiol. 95 (8) (2005) 948-954, http://dx.doi.org/10.1016/j.amjcard.2004.12.032.

[10] T. Omland, J.A. de Lemos, M.S. Sabatine, C.A. Christophi, M.M. Rice, K.A. Jablonski, S. Tjora, M.J. Domanski, B.J. Gersh, J.L. Rouleau, M.A. Pfeffer, E.I. Braunwald, Prevention of events with angiotensin converting enzyme inhibition trial, a sensitive cardiac troponin T assay in stable coronary artery disease, N. Engl. J. Med. 361 (26) (2009) 2538-2547, http://dx.doi.org/10.1056/NEJMoa0805299.

[11] E. Berendes, C. Schmidt, H. Van Aken, M.G. Hartlage, M. Rothenburger, S. Wirtz, H.H. Scheld, G. Brodner, M. Walter, A-type and B-type natriuretic peptides in cardiac surgical procedures, Anesth. Analg. 98 (1) (2004) 11-19, http://dx.doi.org/10. 1213/01.ANE.0000093249.35075.F1.

[12] A.A. Fox, J.D. Muehlschlegel, S.C. Body, S.K. Shernan, K.Y. Liu, T.E. Perry, S.F. Aranki, E.F. Cook, E.R. Marcantonio, C.D. Collard, Comparison of the utility of preoperative versus postoperative B-type natriuretic peptide for predicting hospital length of stay and mortality after primary coronary artery bypass grafting, Anesthesiology 112 (4) (2010) 842-851, http://dx.doi.org/10.1097/ALN. 0b013e3181d23168.

[13] R. Hutfless, R. Kazanegra, M. Madani, M.A. Bhalla, A. Tulua-Tata, A. Chen, P. Clopton, C. James, A. Chiu, A.S. Maisel, Utility of B-type natriuretic peptide in predicting postoperative complications and outcomes in patients undergoing heart surgery, J. Am. Coll. Cardiol. 43 (10) (2004) 1873-1879, http://dx.doi.org/10. 1016/j.jacc.2003.12.048

[14] C.L. Soraas, C. Friis, K.V. Engebretsen, L. Sandvik, S.E. Kjeldsen, T. Tonnessen, Troponin $\mathrm{T}$ is a better predictor than creatine kinase-MB of long-term mortality after coronary artery bypass graft surgery, Am. Heart J. 164 (5) (2012) 779-785, http://dx.doi.org/10.1016/j.ahj.2012.05.027.

[15] J. Holm, M. Vidlund, F. Vanky, O. Friberg, E. Hakanson, S. Walther, R. Svedjeholm, EuroSCORE II and N-terminal pro-B-type natriuretic peptide for risk evaluation: an observational longitudinal study in patients undergoing coronary artery bypass graft surgery, Br. J. Anaesth. 113 (1) (2014) 75-82, http://dx.doi.org/10.1093/bja/ aeu088.
[16] L. Petaja, H. Rosjo, L. Mildh, R. Suojaranta-Ylinen, K.M. Kaukonen, J.J. Jokinen, M. Salmenpera, T.A. Hagve, T. Omland, V. Pettila, Predictive value of high-sensitivity troponin $\mathrm{T}$ in addition to EuroSCORE II in cardiac surgery, Interact. Cardiovasc. Thorac. Surg. 23 (1) (2016) 133-141, http://dx.doi.org/10.1093/icvts/ ivw060.

[17] Y. Bai, L. Wang, Z. Guo, Q. Chen, N. Jiang, J. Dai, J. Liu, Performance of EuroSCORE II and SinoSCORE in Chinese patients undergoing coronary artery bypass grafting, Interact. Cardiovasc. Thorac. Surg. 23 (5) (2016) 733-739, http://dx doi.org/10.1093/icvts/ivw224.

[18] R.L. Osnabrugge, A.M. Speir, S.J. Head, C.E. Fonner, E. Fonner, A.P. Kappetein, J.B. Rich, Performance of EuroSCORE II in a large US database: implications for transcatheter aortic valve implantation, Eur. J. Cardiothorac. Surg. 46 (3) (2014) 400-408 discussion 408 https://doi.org/10.1093/ejcts/ezu033.

[19] S. Nisula, K.M. Kaukonen, S.T. Vaara, A.M. Korhonen, M. Poukkanen, S. Karlsson, M. Haapio, O. Inkinen, I. Parviainen, R. Suojaranta-Ylinen, J.J. Laurila, J. Tenhunen, M. Reinikainen, T. Ala-Kokko, E. Ruokonen, A. Kuitunen, V. Pettila, Incidence, risk factors and 90-day mortality of patients with acute kidney injury in Finnish intensive care units: the FINNAKI study, Intensive Care Med. 39 (3) (2013) 420-428, http://dx.doi.org/10.1007/s00134-012-2796-5.

[20] R. Linko, M. Okkonen, V. Pettila, J. Perttila, I. Parviainen, E. Ruokonen, J. Tenhunen, T. Ala-Kokko, T. Varpula, F.I.-s. group, Acute respiratory failure in intensive care units. FINNALI: a prospective cohort study, Intensive Care Med. 35 (8) (2009) 1352-1361, http://dx.doi.org/10.1007/s00134-009-1519-z.

[21] T. Mueller, A. Gegenhuber, B. Dieplinger, W. Poelz, M. Haltmayer, Long-term stability of endogenous B-type natriuretic peptide (BNP) and amino terminal proBNP (NT-proBNP) in frozen plasma samples, Clin. Chem. Lab. Med. 42 (8) (2004) 942-944, http://dx.doi.org/10.1515/CCLM.2004.153.

[22] W.L. Nowatzke, T.G. Cole, Stability of N-terminal pro-brain natriuretic peptide after storage frozen for one year and after multiple freeze-thaw cycles, Clin. Chem. 49 (9) (2003) 1560-1562.

[23] M. Mansour, L. Clark, P.A. Kavsak, Effect of freeze-thaw and refrigeration conditions on high-sensitivity troponin T concentrations, Ann. Clin. Biochem. 49 (Pt 1) (2012) 101-102, http://dx.doi.org/10.1258/acb.2011.011204.

[24] Roche Diagnostics Limited, Elecsys ${ }^{\circledR}$ Troponin T high sensitive (TnT-hs), http:// www.cobas.com/home/product/clinical-and-immunochemistry-testing/elecsystroponin-t-hs-tnt-hs.html, (2015) (Accessed 13 February 2017).

[25] F.S. Apple, P.O. Collinson, Ifcc Task Force on Clinical Applications of Cardiac Biomarkers, Analytical characteristics of high-sensitivity cardiac troponin assays, Clin. Chem. 58 (1) (2012) 54-61, http://dx.doi.org/10.1373/clinchem.2011. 165795.

[26] M. Okkonen, M. Varpula, R. Linko, J. Perttila, T. Varpula, V. Pettila, F.S. Group, Nterminal-pro-BNP in critically ill patients with acute respiratory failure: a prospective cohort study, Acta Anaesthesiol. Scand. 55 (6) (2011) 749-757, http://dx doi.org/10.1111/j.1399-6576.2011.02439.x.

[27] G. Biondi-Zoccai, E. Romagnoli, P. Agostoni, D. Capodanno, D. Castagno, F. D'Ascenzo, G. Sangiorgi, M.G. Modena, Are propensity scores really superior to standard multivariable analysis? Contemporary Clinical Trials 32 (5) (2011) 731-740, http://dx.doi.org/10.1016/j.cct.2011.05.006.

[28] O.V. Demler, M.J. Pencina, R.B. D'Agostino Sr., Misuse of DeLong test to compare AUCs for nested models, Stat. Med. 31 (23) (2012) 2577-2587, http://dx.doi.org/ $10.1002 / \operatorname{sim} .5328$.

[29] M.J. Pencina, R.B. D'Agostino Sr., E.W. Steyerberg, Extensions of net reclassification improvement calculations to measure usefulness of new biomarkers, Stat. Med. 30 (1) (2011) 11-21, http://dx.doi.org/10.1002/sim.4085.

[30] R. Tibshirani, Regression shrinkage and selection via the lasso, J. R. Stat. Soc. Ser. B Methodol. 58 (1) (1996) 267-288.

[31] B.H. Cuthbertson, B.L. Croal, D. Rae, K. Harrild, P.H. Gibson, G.J. Prescott, A.P. Kengne, G.S. Hillis, N-terminal pro-B-type natriuretic peptide concentrations and long-term outcome after cardiac surgery: a prospective cohort study, Br. J. Anaesth. 110 (2) (2013) 214-221, http://dx.doi.org/10.1093/bja/aes379.

[32] I.K. Toumpoulis, C.E. Anagnostopoulos, J.J. DeRose, D.G. Swistel, European system for cardiac operative risk evaluation predicts long-term survival in patients with coronary artery bypass grafting, Eur. J. Cardiothorac. Surg. 25 (1) (2004) 51-58.

[33] N. Ad, S.D. Holmes, J. Patel, G. Pritchard, D.J. Shuman, L. Halpin, Comparison of EuroSCORE II, original EuroSCORE, and the Society of Thoracic Surgeons risk score in cardiac surgery patients, Ann. Thorac. Surg. 102 (2) (2016) 573-579, http://dx. doi.org/10.1016/j. athoracsur.2016.01.105.

[34] T.M. Kieser, M.S. Rose, S.J. Head, Comparison of logistic EuroSCORE and EuroSCORE II in predicting operative mortality of 1125 total arterial operations, Eur. J. Cardiothorac. Surg. (2016), http://dx.doi.org/10.1093/ejcts/ezw072.

[35] M. Poullis, M. Pullan, J. Chalmers, N. Mediratta, The validity of the origina EuroSCORE and EuroSCORE II in patients over the age of seventy, Interact. Cardiovasc. Thorac. Surg. 20 (2) (2015) 172-177, http://dx.doi.org/10.1093/icvts/ ivu345.

[36] E. Duceppe, J. Parlow, P. MacDonald, K. Lyons, M. McMullen, S. Srinathan, M. Graham, V. Tandon, K. Styles, A. Bessissow, D.I. Sessler, G. Bryson, P.J. Devereaux, Canadian cardiovascular society guidelines on perioperative cardiac risk assessment and management for patients who undergo noncardiac surgery, Can. J. Cardiol. 33 (1) (2017) 17-32, http://dx.doi.org/10.1016/j.cjca.2016.09. 008.

[37] P. Ponikowski, A.A. Voors, S.D. Anker, H. Bueno, J.G. Cleland, A.J. Coats, V. Falk, J.R. Gonzalez-Juanatey, V.P. Harjola, E.A. Jankowska, M. Jessup, C. Linde, P. Nihoyannopoulos, J.T. Parissis, B. Pieske, J.P. Riley, G.M. Rosano, L.M. Ruilope, F. Ruschitzka, F.H. Rutten, P. van der Meer, ESC Guidelines for the diagnosis and treatment of acute and chronic heart failure, Eur. J. Heart Fail. 18 (8) (2016) 891-975, http://dx.doi.org/10.1002/ejhf.592.

[38] D.A. Morrow, J.A. de Lemos, Benchmarks for the assessment of novel cardiovascular biomarkers, Circulation 115 (8) (2007) 949-952, http://dx.doi.org/10.1161/ CIRCULATIONAHA.106.683110. 